\title{
Asymptotic Oscillator Tracking Performance Analysis for Distributed Massive MIMO Systems
}

\author{
D. Richard Brown III, Rui Wang \\ Dept. of Electrical and Computer Eng. \\ Worcester Polytechnic Institute \\ 100 Institute Rd, Worcester, MA 01609 \\ Email: $\{$ drb,rwang@wpi.edu\}
}

\author{
Soura Dasgupta \\ Dept. of Electrical and Computer Engineering \\ University of Iowa \\ Iowa City, IA 52242 \\ Email: dasgupta@engineering.uiowa.edu
}

\begin{abstract}
This paper considers the problem of estimating and tracking channels in a distributed transmission system with $N_{t}$ transmit nodes and $N_{r}$ receive nodes. Since each node in the distributed transmission system has an independent local oscillator, the effective channel between each transmit node and each receive node has time-varying phase and frequency offsets which much be tracked and predicted to facilitate coherent transmission. This paper presents a unified state-space model in which a single node aggregates all of the observations and tracks all of the channel states in the system. To quantify the performance of this tracking system, the steady-state prediction covariance is analyzed. Since the dimension of the prediction covariance matrix grows proportionally to the product $N_{t} N_{r}$, the resulting discrete-time algebraic Riccati equation is difficult to solve for large systems, e.g., distributed massive MIMO systems. It is shown, however, that the structure of the dynamic model allows the steady-state prediction covariance to be efficiently calculated irrespective of the number of transmit and receive nodes. An asymptotic analysis is also presented for systems with a large number of transmit and receive nodes with closed-from results for all of the elements in the asymptotic prediction covariance as a function of the carrier frequency, oscillator parameters, and channel measurement period. Numeric results confirm the analysis and demonstrate the effect of the oscillator parameters on the ability of the distributed transmission system to achieve coherent transmission.
\end{abstract}

Index Terms—oscillator dynamics, distributed communication systems, coherent transmission, channel prediction, distributed beamforming

\section{INTRODUCTION}

We consider the distributed multi-input multi-output (MIMO) communication scenario in Fig. 1 where a transmission cluster with $N_{t}$ transmit nodes communicates with a receive cluster with $N_{r}$ receive nodes. The transmit cluster uses coherent transmission techniques, e.g., distributed transmit beamforming [1]-[5] and/or distributed transmit nullforming [6]-[8], to communicate with the receive cluster. The receivers facilitate coherent transmission by estimating the channels and providing feedback to the transmit cluster.

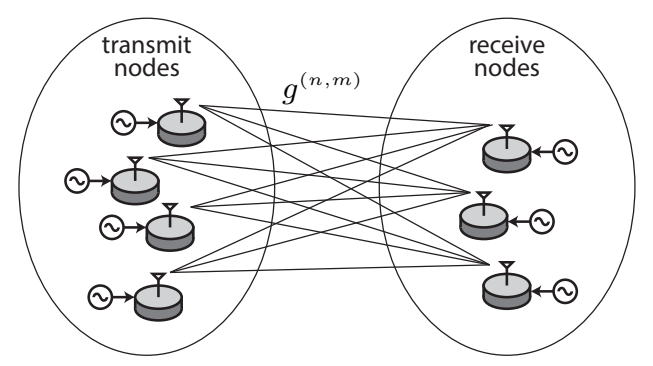

Fig. 1. Distributed MIMO system model with $N_{t}$ transmit nodes and $N_{r}$ receive nodes. Each node possesses a single antenna and an independent oscillator.

This work was supported by the National Science Foundation awards CCF1302104 and CCF-1319458.
Since each node in the distributed transmission system has an independent local oscillator, the effective channel between each transmit node and each receive node has time-varying phase and frequency offsets which much be tracked and predicted to facilitate coherent transmission. Several recent papers have analyzed the performance of distributed beamforming and distributed nullforming subject to independent oscillator dynamics [5], [8]-[10]. The early work in this area made the simplifying assumption that each received individually tracked tracked its own $N_{t}$ channels. More recently, the idea of "unified" tracking has been studied in which a single transmit or receive node aggregates all of the observations and tracks all $N_{t} N_{r}$ channels in the system [10]. A system with unified tracking achieves optimal performance by exploiting the correlations in the states across receive nodes.

This paper presents a performance analysis of unified channel tracking for distributed MIMO systems for the case where the propagation channels $g^{(n, m)}$ are time-invariant (or slowly-varying with respect to the oscillator dynamics) and the stochastic oscillator parameters are identical for all of the nodes in the system. Specifically, we analyze the steady-state prediction covariance of the unified tracking system since the steady-state prediction covariance is directly related to the achievable beamforming and nullforming performance [10], [11]. Since the dimension of the prediction covariance matrix grows proportionally to the product $N_{t} N_{r}$, the resulting discretetime algebraic Riccati equation is difficult to solve for large systems, e.g., distributed massive MIMO systems [12], [13]. We show, however, that the structure of the unified state-space model allows the steady-state prediction covariance to be efficiently calculated irrespective of the number of transmit and receive nodes. We also present an asymptotic analysis for distributed massive MIMO systems with $N_{t} \rightarrow \infty$ and $N_{r}=\eta N_{t}$, and develop closed-from results for all of the elements in the asymptotic prediction covariance as a function of the carrier frequency, oscillator parameters, and channel measurement period. Numeric results confirm the analysis and demonstrate the effect of the oscillator parameters on the ability of the distributed transmission system to achieve coherent transmission.

\section{SYSTEM MODEL}

Each node in the system shown in Fig. 1 is assumed to possess a single antenna. The nominal transmit frequency in the forward link from the distributed transmit cluster to the receivers is at $\omega_{c}$. All forward link channels are modeled as narrowband, linear, and time invariant (LTI). We denote the channel from transmit node $n$ to receive node $m$ at carrier frequency $\omega_{c}$ as $g^{(n, m)} \in \mathbb{C}$ for transmit node $n=1, \ldots, N_{t}$ and receive node $m=1, \ldots, N_{r}$. These LTI propagation channels, in contrast to the time-varying "effective" 
channels described below, do not include the effect of carrier phase offsets between transmit node $n$ and receive node $m$.

The receivers in the system periodically measure the channels from the transmit cluster and provide their measurements to a single node (either a transmit node or a receive node) for unified tracking as in [10]. Fig. 2 shows the effective narrowband channel model from transmit node $n$ to receive node $m$ which includes the effects of propagation and carrier offset. Transmissions $n \rightarrow m$ are conveyed on a carrier nominally at $\omega_{c}$ generated at transmit node $n$, incur a phase shift of $\psi^{(n, m)}=\angle g^{(n, m)}$ over the wireless channel, and are then downmixed by receive node $m$ using its local carrier nominally at $\omega_{c}$. At time $\tau$, the effective narrowband channel from transmit node $n$ to receive node $m$ is modeled as

$$
h^{(n, m)}(\tau)=g^{(n, m)} e^{j\left(\phi_{t}^{(n)}(\tau)-\phi_{r}^{(m)}(\tau)\right)}=\left|g^{(n, m)}\right| e^{j \phi^{(n, m)}(\tau)}
$$

where $\phi_{t}^{(n)}(\tau)$ and $\phi_{r}^{(m)}(\tau)$ are the local carrier phase offsets at transmit node $n$ and receive node $m$, respectively, at time $\tau$ with respect to an ideal carrier reference, and $\phi^{(n, m)}(\tau)=\phi_{t}^{(n)}(\tau)-$ $\phi_{t}^{(m)}(\tau)+\psi^{(n, m)}$ is the pairwise phase offset after propagation between transmit node $n$ and receive node $m$ at time $\tau$.

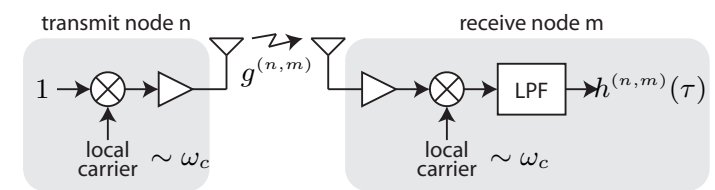

Fig. 2. Effective narrowband channel model including the effects of propagation, transmit and receive gains, and carrier offset.

\section{A. Oscillator Dynamics}

Every node in the system is assumed to have an independent local oscillator. These local oscillators have inherent frequency offsets and behave stochastically, causing phase offset variations in each effective channel from transmit node $n$ to receive node $m$ even when the propagation channels $g^{(n, m)}$ are otherwise time invariant. This section describes a discrete-time dynamic model to characterize the dynamics of the phase variations in $h^{(n, m)}(\tau)$.

Based on the two-state models in [14], [15], we define the discrete-time state of the $n^{\text {th }}$ transmit node's carrier as $x_{t}^{(n)}[k]=$ $\left[\phi_{t}^{(n)}[k], \dot{\phi}_{t}^{(n)}[k]\right]^{\top}$ where $\phi_{t}^{(n)}[k]$ corresponds to the carrier phase offset in radians at transmit node $n$ with respect to an ideal carrier phase reference. The state update of the $n^{\text {th }}$ transmit node's carrier is then

$$
x_{t}^{(n)}[k+1]=f(T) x_{t}^{(n)}[k]+u_{t}^{(n)}[k] \text { with } f(T)=\left[\begin{array}{ll}
1 & T \\
0 & 1
\end{array}\right]
$$

where $T>0$ is the state update period. The process noise vector $u_{t}^{(n)}[k] \stackrel{\text { i.i.d. }}{\sim} \mathcal{N}\left(0, Q_{t}^{(n)}(T)\right)$ causes the carrier derived from the local oscillator at transmit node $n$ to deviate from an ideal linear phase trajectory. The covariance of the discrete-time process noise is derived from a continuous-time model in [14] and is

$$
Q_{t}^{(n)}(T)=\omega_{c}^{2} T\left[\begin{array}{cc}
\alpha_{t}^{(n)}+\beta_{t}^{(n)} \frac{T^{2}}{3} & \beta_{t}^{(n)} \frac{T}{2} \\
\beta_{t}^{(n)} \frac{T}{2} & \beta_{t}^{(n)}
\end{array}\right]
$$

where $\omega_{c}$ is the nominal common carrier frequency in radians per second and $\alpha_{t}^{(n)}$ (units of seconds) and $\beta_{t}^{(n)}$ (units of Hertz) are the process noise parameters corresponding to white frequency noise and random walk frequency noise, respectively. The process noise parameters $\alpha_{t}^{(n)}$ and $\beta_{t}^{(n)}$ can be estimated by fitting the theoretical
Allan variance $\sigma_{y}^{2}(\tau)=\frac{p_{t}^{(n)}}{\tau}+\frac{q_{t}^{(n)} \tau}{3}$ to experimental measurements of the Allan variance over a range of $\tau$ values. For example, a least squares fit to the Allan variance specifications for a Rakon RPFO45 oven-controlled oscillator [16] yields $\alpha_{t}^{(n)}=2.31 \times 10^{-21}$ and $\beta_{t}^{(n)}=6.80 \times 10^{-23}$.

The receive nodes in the system also have independent local oscillators used to generate carriers for downmixing that are governed by the same dynamics as (2) with state $x_{r}^{(m)}[k]$, process noise $u_{r}^{(m)}[k] \stackrel{\text { i.i.d. }}{\sim} \mathcal{N}\left(0, Q_{r}^{(m)}(T)\right)$, and process noise parameters $\alpha_{r}^{(m)}$ and $\beta_{r}^{(m)}$ as in (3) for $m=1, \ldots, N_{r}$.

Since receive nodes can only measure the relative phase and frequency of the transmit nodes after propagation, we define the pairwise offset after propagation as

$$
\delta^{(n, m)}[k]=\left[\begin{array}{l}
\phi^{(n, m)}[k] \\
\dot{\phi}^{(n, m)}[k]
\end{array}\right]=x_{t}^{(n)}[k]+\left[\begin{array}{c}
\psi^{(n, m)} \\
0
\end{array}\right]-x_{r}^{(m)}[k] .
$$

Note that $\delta^{(n, m)}[k]$ is governed by the state update

$$
\delta^{(n, m)}[k+1]=f(T) \delta^{(n, m)}[k]+u_{t}^{(n)}[k]-u_{r}^{(m)}[k] .
$$

We assume that observations are so short as to only provide useful phase estimates. An observation of the $n \rightarrow m$ channel at receive node $m$ is then $y^{(n, m)}[k]=h \delta^{(n, m)}[k]+v^{(n, m)}[k]$ where $h=[1,0]$ and $v^{(n, m)}[k] \stackrel{\text { i.i.d. }}{\sim} \mathcal{N}(0, r)$ is the measurement noise which is assumed to be spatially and temporally i.i.d., and independent of the process noise.

\section{B. Unified State-Space Model}

We assume that a single transmit or receive node aggregates all of the observations and tracks all of the pairwise offset states in the system. The $2 N_{t} N_{r}$-dimensional vector state of pairwise offsets is defined as $\delta[k]=\left[\left(\delta^{(1)}[k]\right)^{\top}, \ldots,\left(\delta^{\left(N_{r}\right)}[k]\right)^{\top}\right]^{\top}$ with $\delta^{(m)}[k]=$ $\left[\left(\delta^{(1, m)}[k]\right)^{\top}, \ldots,\left(\delta^{\left(N_{t}, m\right)}[k]\right)^{\top}\right]^{\top}$. The state update follows as

$$
\begin{aligned}
\delta[k+1] & =\left[\begin{array}{ccc}
f(T) & & \\
& \ddots & \\
& & f(T)
\end{array}\right] \delta[k]+\left[\begin{array}{c}
u_{t}^{(1)}[k]-u_{r}^{(1)}[k] \\
\vdots \\
u_{t}^{\left(N_{t}\right)}[k]-u_{r}^{\left(N_{r}\right)}[k]
\end{array}\right] \\
& =F(T) \delta[k]+G u[k]
\end{aligned}
$$

with the process noise vector $u[k]$ = $\left[\left(u_{t}^{(1)}[k]\right)^{\top}, \ldots,\left(u_{t}^{\left(N_{t}\right)}[k]\right)^{\top},\left(u_{r}^{(1)}[k]\right)^{\top}, \ldots,\left(u_{r}^{\left(N_{r}\right)}[k]\right)^{\top}\right]^{\top} \in$ $\mathbb{R}^{2\left(N_{t}+N_{r}\right)}$ and

$$
G=\left[\begin{array}{cccc}
I_{2 N_{t}} & J_{2 N_{t}} & & \\
\vdots & & \ddots & \\
I_{2 N_{t}} & & & J_{2 N_{t}}
\end{array}\right] \in \mathbb{R}^{2 N_{t} N_{r} \times 2\left(N_{t}+N_{r}\right)}
$$

where $I_{n}$ denotes an $n \times n$ identity matrix and $J_{2 N_{t}}=$ $-\left[I_{2}, \ldots, I_{2}\right]^{\top} \in \mathbb{R}^{2 N_{t} \times 2}$. The $N_{t} N_{r}$-dimensional vector observation is then

$$
\begin{aligned}
y[k] & =\left[\begin{array}{lll}
h & & \\
& \ddots & \\
& & h
\end{array}\right] \delta[k]+v[k] \\
& =H \delta[k]+v[k]
\end{aligned}
$$

where $H \in \mathbb{R}^{N_{t} N_{r} \times 2 N_{t} N_{r}} \quad$ and $v[k]=$ $\left[v^{(1,1)}[k], \ldots, v^{\left(N_{t}, N_{r}\right)}[k]\right]^{\top} \in \mathbb{R}^{N_{t} N_{r}}$ is i.i.d. measurement noise. 


\section{Discussion}

Note that the state update (5) specifies a dynamic system where the states are coupled only through the correlated process noise. The process noise is correlated through all of the receive oscillators as shown in (6). While the number of states grows according to the product $N_{t} N_{r}$, the number of independent oscillators grows according to the sum $N_{t}+N_{r}$. In fact, if we assume the transmit and receive nodes have identical and independent process noise statistics with $Q_{t}^{(n)}(T)=Q_{r}^{(m)}(T)=q$, then $\mathrm{E}\left[u[k] u^{\top}[k]\right]=I_{N_{t}+N_{r}} \otimes q$ and process noise covariance can be written as

$$
\begin{aligned}
& Q=G \mathrm{E}\left[u[k] u^{\top}[k]\right] G^{\top}
\end{aligned}
$$

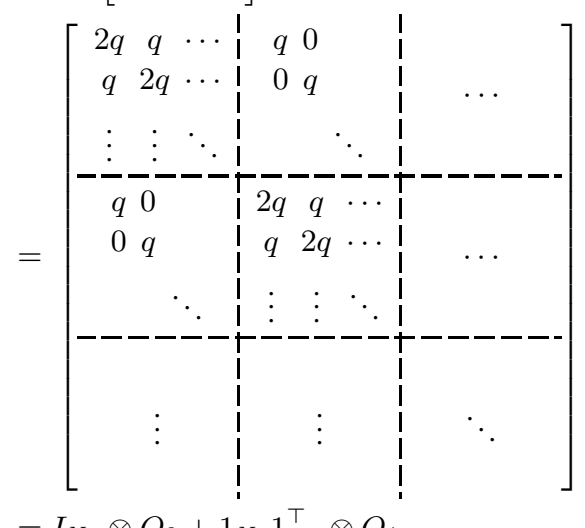

$$
\begin{aligned}
& =I_{N_{r}} \otimes Q_{0}+1_{N_{r}} 1_{N_{r}}^{\top} \otimes Q_{1}
\end{aligned}
$$

with $Q_{0}=1_{N_{t}} 1_{N_{t}}^{\top} \otimes q$ and $Q_{1}=I_{N_{t}} \otimes q$.

Additionally, using the usual tests, one can straightforwardly verify that the system specified in (5) and (7) is completely observable but not stabilizable. Since the system is not stabilizable, most of the results regarding the existence and uniqueness of a steady-state limiting solution to the prediction covariance of the optimal filter do not directly apply. In the following section, we numerically demonstrate that the optimal filter can converge to a stable limiting value and then formalize the existence of such a solution in Section IV.

\section{TRACKING EXAMPLE}

In this section, we numerically demonstrate the convergence of the phase and frequency prediction variance of a Kalman filter tracker for the unified model specified in (5) and (7) for two different systems: $\left\{N_{t}, N_{r}\right\}=\{4,2\}$ and $\left\{N_{t}, N_{r}\right\}=\{32,16\}$. The state update interval was set to $T=0.250$ seconds and the carrier frequency was set to $\omega_{c}=2 \pi \cdot 900 \cdot 10^{6} \mathrm{radians} / \mathrm{sec}$. The process noise parameters set to $\alpha=2.31 \times 10^{-21}$ seconds and $\beta=6.80 \times 10^{-23}$ Hertz according to the Rakon RPFO45 ovencontrolled oscillator parameters in Section II-A. The measurement noise variance was set to $r=(2 \pi \cdot 10 / 360)^{2} \operatorname{rad}^{2}$.

The initial prediction covariance, i.e., $P[0]=\mathrm{E}\left[\delta[0] \delta^{\top}[0]\right]$ was determined based on the following assumptions:

1) Initial oscillator phases $x_{t}^{(n)}[0]$ and $x_{r}^{(m)}[0]$ are i.i.d. uniformly distributed on $[-\pi, \pi]$.

2) Channel phases $\psi^{(n, m)}$ are i.i.d. uniformly uniformly distributed on $[-\pi, \pi]$.

3) Initial frequency offsets are i.i.d. uniformly distributed on $[-\nu, \nu]$ where $\nu=\mathrm{ppm} \cdot 10^{-6} \cdot 2 \pi f_{c}$ and where "ppm" refers to the oscillators' "parts-per-million" frequency accuracy specification.

All of these random variables are further assumed to be independent.
The prediction covariance at time $k+1$ satisfies [17]

$P[k+1]=F\left(P[k]-P[k] H^{\top}\left(H P[k] H^{\top}+R\right)^{-1} H P[k]\right) F^{\top}+Q$.

Figure 3 plots the $(1,1)$ and $(2,2)$ elements of the prediction covariance matrix, corresponding to the phase prediction variance and frequency prediction variance, respectively, versus the experimentally determined prediction variances obtained via Monte-Carlo simulation of the Kalman filter.
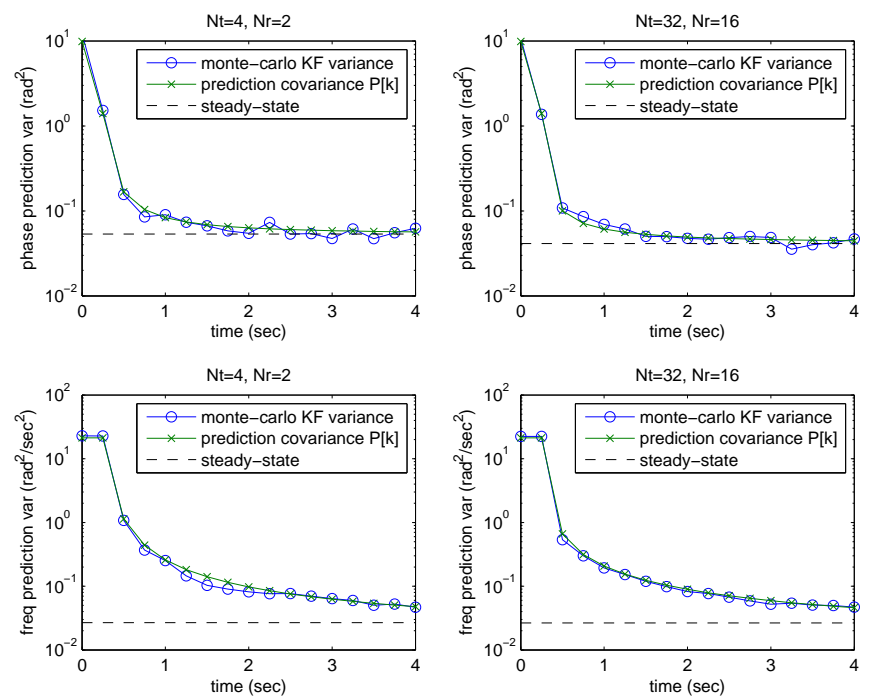

Fig. 3. Phase and frequency prediction variances for a Kalman filter tracker of the unified state-space model.

These examples show that the phase and frequency prediction variances of the Kalman filter converge toward their steady-state predictions which were obtained by solving a discrete-time algebraic Riccati equation. Moreover, the steady-state phase prediction variance (which tends to be a good indicator of the potential beamforming or nullforming performance in distributed MIMO systems [10]) for the larger system with $N_{t}=32$ and $N_{r}=16$ is somewhat better than the steady-state phase prediction variance for the smaller system with $N_{t}=4$ and $N_{r}=2$. The following section formalizes the existence of the steady-state prediction covariance in the unified tracking model and develops closed-form expressions for the asymptotic prediction covariance as $N_{t} \rightarrow \infty$ with $N_{r}=\eta N_{t}$.

\section{Steady-State Prediction Covariance Analysis}

In this section, we analyze the steady-state behavior of a Kalman filter tracker for the unified state $\delta[k]$. In steady-state, the prediction covariance $P$ of the Kalman filter will satisfy

$$
P=F\left[P-P H^{\top}\left(H P H^{\top}+R\right)^{-1} H P\right] F^{\top}+Q .
$$

This is a version of the well-known discrete-time algebraic Riccati equation (DARE) which has a unique symmetric positive semidefinite solution when $[F, H]$ is a detectable pair and $\left[F, Q^{1 / 2}\right]$ is a stabilizable pair [17]. The system specified in (5) and (7) does not satisfy these conditions, however, since it can be shown that $\left[F, Q^{1 / 2}\right]$ is not stabilizable. Nevertheless, it turns out that we can still find a symmetric positive semidefinite solution to (9) for the unified tracking model as shown below.

The following theorem establishes that complete observability is sufficient for there to exist a symmetric positive semidefinite solution to (9). 
Theorem 1. Suppose $[F, H]$ is completely observable. Then (9) has a symmetric positive semidefinite solution.

A proof of this theorem will be provided in a expanded journal paper. Intuitively, one can perform a similarity transformation to put the system into a "controllability staircase form" [18, p.159]. Part of the transformed system is then detectable and stabilizable and the positive semidefinite steady-state prediction covariance for this part of the transformed system is uniquely determined [17]. The remaining prediction covariances of the transformed system can be set to zero and it can be shown that this solution satisfies (9).

For the system specified in (5) and (7), $P$ has dimensions $2 N_{t} N_{r} \times$ $2 N_{t} N_{r}$. It can be computationally difficult to solve (9) for large $N_{t}$ and/or large $N_{r}$ since the dimensions of the similarity transform become large and the dimension of the resulting reduced-dimensional DARE still grows without bound as $N_{t} \rightarrow \infty$ and/or $N_{r} \rightarrow \infty$. Nevertheless, as we will show below, (9) can be efficiently solved when the $F, H, R$, and $Q$ matrices have a certain structure.

We first establish some notation and a useful preliminary result. For any matrices $A$ and $B$ with the same dimension, we denote

$$
\begin{aligned}
\Gamma_{n}(A, B) & =I_{n} \otimes A+1_{n} 1_{n}^{\top} \otimes B \\
& =\left[\begin{array}{cccc}
A+B & B & \cdots & B \\
B & A+B & & \\
\vdots & & \ddots & \\
B & & & A+B
\end{array}\right] .
\end{aligned}
$$

Note that $Q$ in (8) can be written as $Q=\Gamma_{N_{r}}\left(Q_{0}, Q_{1}\right)$ using this notation.

Denoting the distinct eigenvalues of a square matrix $C$ as $\lambda(C)$, the following lemma relates the eigenvalues of $\Gamma_{n}(A, B)$ to the eigenvalues of $A$ and $A+n B$ when $A$ and $B$ are square matrices.

Lemma 1. Given $A \in \mathbb{R}^{s \times s}$ and $B \in \mathbb{R}^{s \times s}$ and $\Gamma_{n}(A, B)$ defined in (10). Then $\lambda(A) \cup \lambda(A+n B)=\lambda\left(\Gamma_{n}(A, B)\right)$.

A proof of this lemma is provided in Appendix A. Note that Lemma 1 implies that if $\Gamma_{n}(A, B)$ is positive semidefinite, then both $A$ and $A+n B$ are positive semidefinite. This result is used in the proof of the following Theorem.

Theorem 2. If there exists a positive semidefinite solution to (9) and

$$
\begin{aligned}
F & =\Gamma_{n}\left(F_{0}, 0\right) \text { with } F_{0} \in \mathbb{R}^{s \times s}, \\
H & =\Gamma_{n}\left(H_{0}, 0\right) \text { with } H_{0} \in \mathbb{R}^{t \times s}, \\
R & =\Gamma_{n}\left(R_{0}, R_{1}\right) \text { with } R_{0} \in \mathbb{R}^{t \times t} \text { and } R_{1} \in \mathbb{R}^{t \times t} \text {, and } \\
Q & =\Gamma_{n}\left(Q_{0}, Q_{1}\right) \text { with } Q_{0} \in \mathbb{R}^{s \times s} \text { and } Q_{1} \in \mathbb{R}^{s \times s}
\end{aligned}
$$

then $P=\Gamma_{n}\left(P_{0}, P_{1}\right)$ with positive semidefinite $P_{0} \in \mathbb{R}^{s \times s}$ satisfying

$$
P_{0}=F_{0}\left[P_{0}-P_{0} H_{0}^{\top}\left(H_{0} P_{0} H_{0}^{\top}+R_{0}\right)^{-1} H_{0} P_{0}\right] F_{0}^{\top}+Q_{0}
$$

and positive semidefinite $\bar{P}=n^{-1} P_{0}+P_{1} \in \mathbb{R}^{s \times s}$ satisfying

$$
\bar{P}=F_{0}\left[\bar{P}-\bar{P} H_{0}^{\top}\left(H_{0} \bar{P} H_{0}^{\top}+\bar{R}\right)^{-1} H_{0} \bar{P}\right] F_{0}^{\top}+\bar{Q}
$$

with $\bar{R}:=n^{-1} R_{0}+R_{1}$ and $\bar{Q}:=n^{-1} Q_{0}+Q_{1}$.

A proof of Theorem 2 will be provided in an expanded journal paper. Note that Lemma 1 ensures that both $P_{0}$ and $\bar{P}$ are positive semidefinite. Intuitively, the approach in the proof of Lemma 1 can be followed to transform the system into $n$ independent subsystems each of which can be solved separately.
Observe that the unified oscillator tracking system in Section II-B satisfies the requirements of Theorem 2 with $n=N_{r}$. The utility of this theorem is that the $2 N_{t} N_{r} \times 2 N_{t} N_{r}$ DARE in (9) can be solved by computing two smaller $2 N_{t} \times 2 N_{t}$ DAREs. While the dimension of these DAREs also grows without bound as $N_{t} \rightarrow \infty$, it turns out that we can further simplify the solution of (9) by observing that we have the additional structure

$$
\begin{aligned}
F_{0} & =\Gamma_{N_{t}}(f, 0) \\
H_{0} & =\Gamma_{N_{t}}(h, 0) \\
R_{0} & =\Gamma_{N_{t}}(r, 0) \\
R_{1} & =0 \\
Q_{0} & =\Gamma_{N_{t}}(0, q) \\
Q_{1} & =\Gamma_{N_{t}}(q, 0)
\end{aligned}
$$

with $f=f(T) \in \mathbb{R}^{2 \times 2}, h \in \mathbb{R}^{1 \times 2}, r \in \mathbb{R}$, and $q=\in \mathbb{R}^{2 \times 2}$ all defined in Section II-B. Hence, Theorem 2 can be recursively applied to the oscillator tracking system to say that $P=\Gamma_{n}\left(P_{0}, P_{1}\right)$ with

$$
\begin{aligned}
& P_{0}=\Gamma_{N_{t}}\left(p_{00}, p_{01}\right) \\
& P_{1}=\Gamma_{N_{t}}\left(p_{10}, p_{11}\right)
\end{aligned}
$$

where $p_{00}, p_{01}, p_{10}$, and $p_{11}$ are all $2 \times 2$ matrices. This result implies that, irrespective of the number of transmit and receive nodes, the $2 N_{t} N_{r} \times 2 N_{t} N_{r}$ prediction covariance in (9) can be efficiently computed for the unified oscillator tracking scenario by solving four $2 \times 2$ DAREs.

We can show that one of these $2 \times 2$ DAREs is trivial to solve in our unified oscillator tracking scenario. Recursively applying Theorem 2 , we can write

$$
p_{00}=f\left[p_{00}-p_{00} h^{\top}\left(h p_{00} h^{\top}+r\right)^{-1} h p_{00}\right] f^{\top}+0 .
$$

The unique solution to this DARE is $p_{00}=0$, which implies that $P_{0}=\Gamma_{N_{t}}\left(0, p_{01}\right)=1_{N_{t}} 1_{N_{t}}^{\top} \otimes p_{01}$. The following section develops closed-form expressions for $p_{10}, p_{01}$, and $p_{11}$ in the asymptotic regime where $N_{t} \rightarrow \infty$ and $N_{r}=\eta N_{t}$.

\section{Asymptotic Prediction Covariance AnAlysis}

Recall that $\bar{P}=N_{r}^{-1} P_{0}+P_{1}, \bar{Q}=N_{r}^{-1} Q_{0}+Q_{1}$, and $\bar{R}=$ $N_{r}^{-1} R_{0}+R_{1}$. As $N_{r} \rightarrow \infty$, we have $\bar{P} \rightarrow P_{1}, \bar{Q} \rightarrow Q_{1}$, and $\bar{R} \rightarrow R_{1}=0$. Hence, (12) becomes

$$
P_{1}=F_{0}\left[P_{1}-P_{1} H_{0}^{\top}\left(H_{0} P_{1} H_{0}^{\top}\right)^{-1} H_{0} P_{1}\right] F_{0}^{\top}+Q_{1} .
$$

Since $Q_{1}=I_{N_{t}} \otimes q, F_{0}=I_{N_{t}} \otimes f$, and $H_{0}=I_{N_{t}} \otimes h$ are all block diagonal matrices, it is straightforward to see that the asymptotic value of $P_{1}$ is also block diagonal. In other words, $P_{1} \rightarrow I_{N_{t}} \otimes$ $p_{10}$ and $p_{11} \rightarrow 0$. Hence, to determine $P_{1}$ for large $N_{r}$, it is only necessary to solve the $2 \times 2$ DARE

$$
p_{10}=f\left[p_{10}-p_{10} h^{\top}\left(h p_{10} h^{\top}\right)^{-1} h p_{10}\right] f^{\top}+q .
$$

Now consider $P_{0}=1_{N_{t}} 1_{N_{t}}^{\top} \otimes p_{01}$. Defining $\bar{p}_{01}=N_{t}^{-1} p_{00}+p_{01}$, we have that $\bar{p}_{01}=p_{01}$ since, as shown previously, $p_{00}=0$ for any $N_{t}$ and $N_{r}$. Theorem 2 implies that $p_{01}$ satisfies

$$
p_{01}=f\left[p_{01}-p_{01} h^{\top}\left(h p_{01} h^{\top}+N_{r}^{-1} r\right)^{-1} h p_{01}\right] f^{\top}+q
$$

which, in the limit as $N_{r} \rightarrow \infty$, becomes identical to (14). Hence, in the asymptotic regime where $N_{t} \rightarrow \infty$ and $N_{r}=\eta N_{t}$, we have 
$p_{01}=p_{10}=p$ with $p$ satisfying the $2 \times 2$ DARE

$$
p=f\left[p-p h^{\top}\left(h p h^{\top}\right)^{-1} h p\right] f^{\top}+q .
$$

In other words, it is only necessary to solve a single $2 \times 2$ DARE to fully characterize the $2 N_{t} N_{r} \times 2 N_{t} N_{r}$ asymptotic prediction covariance matrix $P$.

Summarizing these results, we have $p_{00}=0, p_{11} \rightarrow 0, p_{01} \rightarrow p$, and $p_{10} \rightarrow p$ as $N_{t} \rightarrow \infty$ with $N_{r}=\eta N_{t}$. Hence,

$$
\begin{aligned}
& P_{0} \rightarrow 1_{N_{t}} 1_{N_{t}}^{\top} \otimes p \\
& P_{1} \rightarrow I_{N_{t}} \otimes p
\end{aligned}
$$

and the asymptotic prediction covariance $P=\Gamma_{n}\left(P_{0}, P_{1}\right)$ takes the same form as (8) with $q$ replaced by $p$.

We now compute closed-form expressions for the elements of $p$. We denote

$$
p=\left[\begin{array}{ll}
p(1,1) & p(1,2) \\
p(2,1) & p(2,2)
\end{array}\right]
$$

and, from (3) under the assumption of identical process noise statistics at each receive node, set

$$
q=\omega_{c}^{2} T\left[\begin{array}{cc}
\alpha+\beta \frac{T^{2}}{3} & \beta \frac{T}{2} \\
\beta \frac{T}{2} & \beta
\end{array}\right] .
$$

Some straightforward algebra on (15) yields

$$
p(1,2)=p(2,1)=\omega_{c}^{2} T^{2} \beta\left(\gamma+\frac{1}{2}\right)
$$

with $\gamma:=\sqrt{\frac{1}{12}+\frac{\alpha}{T^{2} \beta}}$. The remaining elements of $p$ follow as

$$
\begin{aligned}
& p(1,1)=\omega_{c}^{2} T^{3} \beta\left(\gamma+\frac{1}{2}\right)^{2} \\
& p(2,2)=\omega_{c}^{2} T \beta(\gamma+1)
\end{aligned}
$$

Note that the asymptotic prediction covariance is not a function of $\eta=\frac{N_{r}}{N_{t}}$. The asymptotic prediction covariance is only a function of the process noise parameters $\alpha$ and $\beta$ as well as the carrier frequency $\omega_{c}$ and the update period $T$. The parameter $\eta$ only affects the rate at which the elements of the prediction covariance matrix approach their asymptotic values.

\section{NUMERICAL RESULTS}

This section presents numerical results confirming the asymptotic analysis in Section V. Since there are only 12 unique elements in the prediction covariance matrix $P$ irrespective of the number of transmit and receive nodes, Table I lists the 12 relevant elements of $P$, their meanings, and their asymptotic values.

Fig. 4 plots elements of the prediction covariance matrix $P$ versus the number of transmit nodes $N_{t}$ with $N_{r}=\eta N_{t}$ and $\eta=0.2$. The simulation parameters are otherwise identical to those in Section III. These results confirm the asymptotic analysis in Section $\mathrm{V}$ and show that asymptotic results can be accurate predictions of many of the elements of the prediction covariance matrix even for small values of $N_{t}$ and $N_{r}$.

Fig. 5 plots the asymptotic phase standard deviation (in degrees) versus oscillator parameters $\alpha$ and $\beta$ for $T=0.250$ seconds and $\omega_{c}=2 \pi \cdot 900 \cdot 10^{6}$ radians/sec. Specifically, this plot shows $\frac{360}{2 \pi} \cdot \sqrt{p(1,1)}$ over a range of typical oscillator parameters with "good XO" and "poor XO" oscillator parameters fitted to a table of typical Allan variances from [19]. These results show that a system using the Rakon oven-controlled oscillators with $T=0.250$ seconds
TABLE I

UNIQUE ELEMENTS OF THE PREDICTION COVARIANCE MATRIX $P$ WITH $n^{\prime} \neq n$ AND $m^{\prime} \neq m$.

\begin{tabular}{r|l}
$P(i, j)$ & Meaning and asymptotic value \\
\hline$P(1,1)$ & Phase variance $\operatorname{cov}\left(\phi^{(n, m)}, \phi^{(n, m)}\right) \rightarrow 2 p(1,1)$ \\
$P(1,2)$ & Phase/freq covariance $\operatorname{cov}\left(\phi^{(n, m)} \dot{\phi}^{(n, m)}\right) \rightarrow 2 p(1,2)$ \\
$P(2,2)$ & Frequency variance $\operatorname{cov}\left(\dot{\phi}^{(n, m)}, \dot{\phi}^{(n, m)}\right) \rightarrow 2 p(2,2)$ \\
\hline$P(3,1)$ & Phase covariance $\operatorname{cov}\left(\phi^{(n, m)}, \phi^{\left(n^{\prime}, m\right)}\right) \rightarrow p(1,1)$ \\
$P(3,2)$ & Phase/freq covariance $\operatorname{cov}\left(\phi^{(n, m)} \dot{\phi}^{\left(n^{\prime}, m\right)}\right) \rightarrow p(1,2)$ \\
$P(4,2)$ & Frequency variance $\operatorname{cov}\left(\dot{\phi}^{(n, m)}, \dot{\phi}^{\left(n^{\prime}, m\right)}\right) \rightarrow p(2,2)$ \\
\hline$P\left(2 N_{t}+1,1\right)$ & Phase covariance $\operatorname{cov}\left(\phi^{(n, m)}, \phi^{\left(n, m^{\prime}\right)}\right) \rightarrow p(1,1)$ \\
$P\left(2 N_{t}+1,2\right)$ & Phase/freq covariance $\operatorname{cov}\left(\phi^{(n, m)} \dot{\phi}^{\left(n, m^{\prime}\right)}\right) \rightarrow p(1,2)$ \\
$P\left(2 N_{t}+2,2\right)$ & Frequency variance $\operatorname{cov}\left(\dot{\phi}^{(n, m)}, \dot{\phi}^{\left(n, m^{\prime}\right)}\right) \rightarrow p(2,2)$ \\
\hline$P\left(2 N_{t}+3,1\right)$ & Phase covariance $\operatorname{cov}\left(\phi^{(n, m)}, \phi^{\left(n^{\prime}, m^{\prime}\right)}\right) \rightarrow 0$ \\
$P\left(2 N_{t}+3,2\right)$ & Phase/freq covariance $\operatorname{cov}\left(\phi^{(n, m)} \dot{\phi}^{\left(n^{\prime}, m^{\prime}\right)}\right) \rightarrow 0$ \\
$P\left(2 N_{t}+4,2\right)$ & Frequency variance $\operatorname{cov}\left(\dot{\phi}^{(n, m)}, \dot{\phi}^{\left(n^{\prime}, m^{\prime}\right)}\right) \rightarrow 0$
\end{tabular}
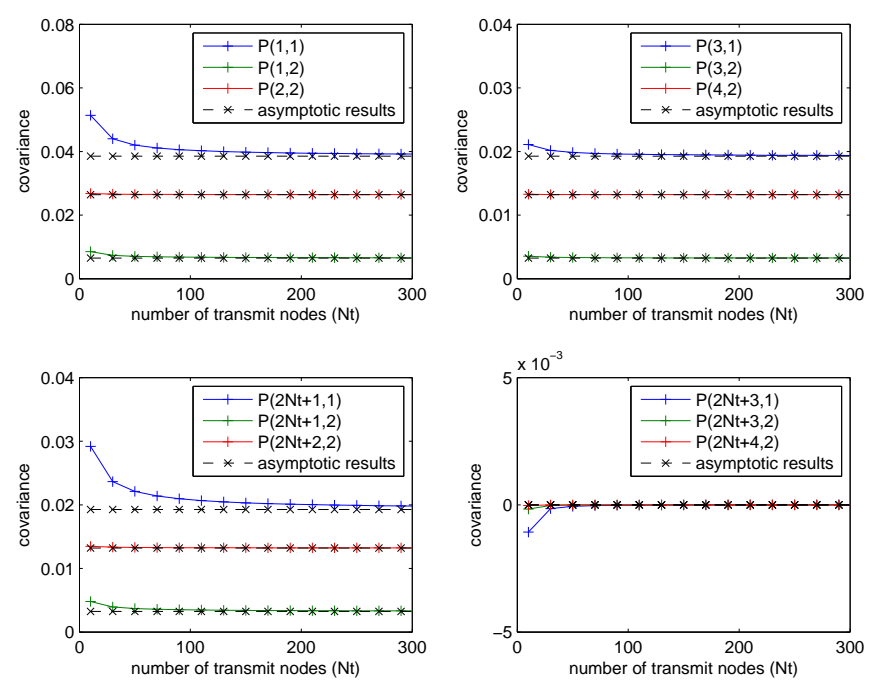

Fig. 4. Relevant elements of the prediction covariance matrix versus the number of transmit nodes $N_{t}$ with $N_{r}=\eta N_{t}$ and $\eta=0.2$

and $\omega_{c}=2 \pi \cdot 900 \cdot 10^{6}$ radians $/ \mathrm{sec}$ will have an asymptotic phase prediction standard deviation of less than 10 degrees, which is more than adequate to achieve good coherent beamforming gains but may be insufficient to achieve deep nulls [10]. The "poor XO" has an asymptotic phase prediction standard deviation so large that coherent distributed transmission is impossible. To achieve coherent transmission with the "poor XO", the carrier frequency $\omega_{c}$ and/or the measurement interval $T$ must be reduced.

\section{CONCLUSION}

This paper analyzed the steady-state prediction covariance of a distributed MIMO system with unified channel tracking and stochastic oscillator dynamics. We showed that a steady-state solution to the discrete-time algebraic Riccati equation exists and the structure of the unified tracking model was exploited to develop efficient solutions for the discrete-time algebraic Riccati equation with low computational complexity not depending on the number of transmit or receive nodes. An asymptotic analysis was also presented for large networks with closed-from results for all of the elements in the asymptotic prediction covariance matrix. Numeric results confirmed the analysis and demonstrated the effect of the oscillator parameters on the ability of the system to achieve coherent transmission. 


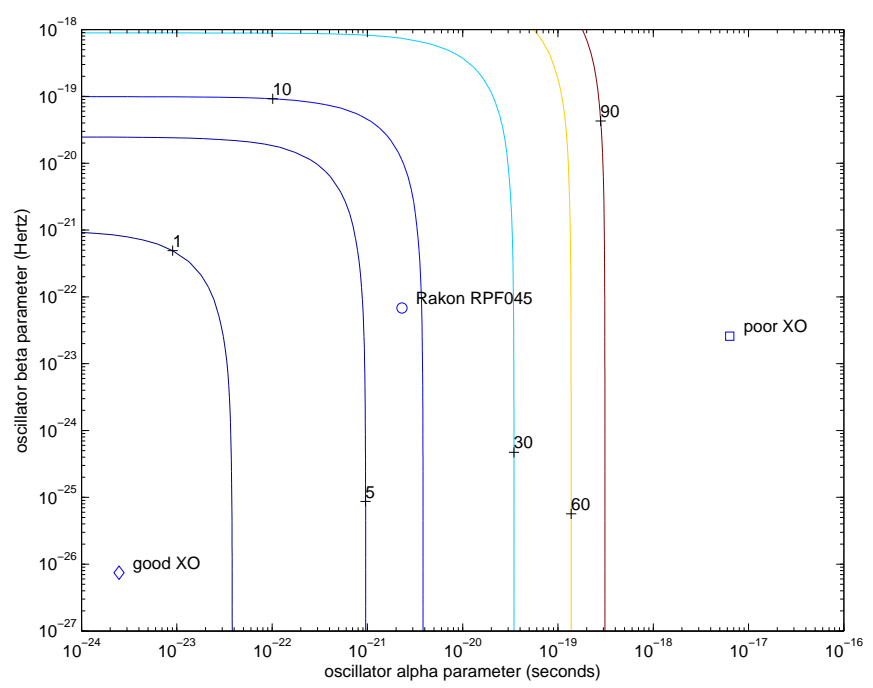

Fig. 5. Asymptotic phase standard deviation (in degrees) versus oscillator parameters $\alpha$ and $\beta$ for $T=0.250$ seconds and $\omega_{c}=2 \pi \cdot 900 \cdot 10^{6}$ radians/sec.

\section{APPENDIX A \\ PROOF OF LEMMA 1}

First consider the matrix $\Gamma_{n}(0,1)=1_{n} 1_{n}^{\top}$. This matrix has an eigenvalue at zero with algebraic multiplicity $n-1$ and an eigenvalue at $n$ corresponding to the eigenvector $1_{n}$. Since $\Gamma_{n}(0,1)$ is real and symmetric, it is diagonalizable and there exists an invertible $T$ such that

$$
T^{-1} \Gamma_{n}(0,1) T=\operatorname{diag}(0, \cdots, 0, n) .
$$

Now let $T_{s}=T \otimes I_{s}$. We can write

$$
\begin{aligned}
T_{s}^{-1} \Gamma_{n}(A, B) T_{s} & =\left(T \otimes I_{s}\right)^{-1}\left(I_{n} \otimes A+1_{n} 1_{n}^{\top} \otimes B\right)\left(T \otimes I_{s}\right) \\
& =\left(T^{-1} \otimes A+T^{-1} 1_{n} 1_{n}^{\top} \otimes B\right)\left(T \otimes I_{s}\right) \\
& =I_{n} \otimes A+\left(T^{-1} 1_{n} 1_{n}^{\top} T\right) \otimes B \\
& =I_{n} \otimes A+(\operatorname{diag}(0, \cdots, 0, n)) \otimes B \\
& =\operatorname{blockdiag}(A, \cdots, A, A+n B)
\end{aligned}
$$

where the second to last equality used (18). Since similarity transformations do not change the eigenvalues, this final result implies that $\lambda(A) \cup \lambda(A+n B)=\lambda\left(\Gamma_{n}(A, B)\right)$.

\section{REFERENCES}

[1] R. Mudumbai, G. Barriac, and U. Madhow, "On the feasibility of distributed beamforming in wireless networks," IEEE Trans. Wireless Commun., vol. 6, no. 5, pp. 1754-1763, May 2007.

[2] R. Mudumbai, D.R. Brown III, U. Madhow, and H.V. Poor, "Distributed transmit beamforming: Challenges and recent progress," IEEE Communications Magazine, vol. 47, no. 2, pp. 102-110, February 2009.

[3] R. Preuss and D.R. Brown III, "Retrodirective distributed transmit beamforming with two-way source synchronization," in Proceedings of the Conference of Information Sciences and Systems (CISS 2010), Princeton, NJ, March 2010, pp. 1-6.

[4] — " "Two-way synchronization for coordinated multi-cell retrodirective downlink beamforming," IEEE Trans. Signal Process., vol. 59, no. 11, pp. 5415-5427, Nov. 2011.

[5] D. Brown, P. Bidigare, and U. Madhow, "Receiver-coordinated distributed transmit beamforming with kinematic tracking," in Acoustics, Speech and Signal Processing (ICASSP), 2012 IEEE International Conference on, Mar. 2012, pp. 5209-5212.

[6] K. Zarifi, S. Affes, and A. Ghrayeb, "Collaborative null-steering beamforming for uniformly distributed wireless sensor networks," IEEE Trans. on Signal Processing, vol. 58, no. 3, pp. 1889 -1903, Mar. 2010.
[7] D.R. Brown III, U. Madhow, S. Dasgupta, and P. Bidigare, "Receivercoordinated distributed transmit nullforming with channel state uncertainty," in Conf. Inf. Sciences and Systems (CISS2012), Mar. 2012.

[8] D.R. Brown III, P. Bidigare, S. Dasgupta, and U. Madhow, "Receivercoordinated zero-forcing distributed transmit nullforming," in Statistical Signal Processing Workshop (SSP), 2012 IEEE, Aug. 2012, pp. 269-272.

[9] D. Brown, R. Mudumbai, and S. Dasgupta, "Fundamental limits on phase and frequency tracking and estimation in drifting oscillators," in Acoustics, Speech and Signal Processing (ICASSP), 2012 IEEE International Conference on, Mar. 2012, pp. 5225-5228.

[10] D. Brown III and R. David, "Receiver-coordinated distributed transmit nullforming with local and unified tracking," in Acoustics, Speech and Signal Processing (ICASSP), 2014 IEEE International Conference on, May 2014.

[11] A. Kumar, R. Mudumbai, S. Dasgupta, M. Rahman, D. Brown III, M. Madhow, and P. Bidigare, "A scalable feedback mechanism for distributed nullforming with phase-only adaptation," Submitted to IEEE Trans. on Signal Processing, currently in review.

[12] J. Hoydis, S. ten Brink, and M. Debbah, "Massive mimo: How many antennas do we need?" in Communication, Control, and Computing (Allerton), 2011 49th Annual Allerton Conference on, 2011, pp. 545550.

[13] E. G. Larsson, F. Tufvesson, O. Edfors, and T. L. Marzetta, "Massive mimo for next generation wireless systems," submitted to IEEE Communications Magazine, vol. abs/1304.6690, 2013.

[14] L. Galleani, "A tutorial on the 2-state model of the atomic clock noise," Metrologia, vol. 45, no. 6, pp. S175-S182, Dec. 2008.

[15] G. Giorgi and C. Narduzzi, "Performance analysis of kalman filterbased clock synchronization in IEEE 1588 networks," in International IEEE Symposium on Precision Clock Synchronization for Measurement, Control, and Communication, October 12-16 2009, pp. 1-6.

[16] "Rakon RFPO45 SMD oven controlled crystal oscillator datasheet," 2009. [Online]. Available: http://www.rakon.com/products/families/download/file?fid=39.133

[17] B. Anderson and J. Moore, Optimal Filtering. Dover, 1979.

[18] C.-T. Chen, Linear System Theory and Design. Oxford University Press, 1999.

[19] W. Klepczynski and P. Ward, "Frequency stability requirements for narrow band receivers," in 32nd Annual Precise Time and Time Interval Meeting, November 2000. 